\title{
Review of checkpoint immunotherapy for the management of non-small cell lung cancer
}

This article was published in the following Dove Press journal: ImmunoTargets and Therapy

\author{
Shine Raju' \\ Ranjit Joseph ${ }^{2}$ \\ Sameep Sehgal ${ }^{3}$ \\ 'Division of Geriatrics and Palliative \\ Care, Case Western University, \\ Cleveland, $\mathrm{OH}, \mathrm{USA} ;{ }^{2}$ Division of \\ Hematology and Oncology, University \\ of North Carolina, Chapel Hill, \\ NC, USA; ${ }^{3}$ Department of Thoracic \\ Medicine and Surgery, Lewis Katz \\ School of Medicine, Temple University, \\ Philadelphia, PA, USA
}

\begin{abstract}
Checkpoint immunotherapy uses highly selective humanized monoclonal antibodies against checkpoint signals such as programmed cell death receptor (PD-1) and programmed cell death ligand (PD-L1). By blocking these receptors and signals, the immune system can be reactivated to fight the tumor. Immunotherapy for advanced non-small cell lung cancer (NSCLC) has resulted in a new paradigm of treatment options resulting in improved survival and response rates and has a less severe yet unique toxicity profile when compared to chemotherapy. PD-1 inhibitors, nivolumab and pembrolizumab, and PD-L1 inhibitor, atezolizumab, are currently approved by regulatory authorities for the treatment of advanced NSCLC. This article provides a detailed review of these newer agents, their mechanism of action, side-effect profile, therapeutic indications and current evidence supporting their use in the management of NSCLC.
\end{abstract}

Keywords: non-small cell lung cancer, PD-1 inhibitors, PD-L1 inhibitors, pembrolizumab, nivolumab, atezolizumab

\section{Introduction}

Lung cancer is the leading cause of cancer-related deaths in the United States among both men and women. It is estimated to have contributed to 222,500 new cases and 158,870 deaths in the US alone in $2017 .{ }^{1}$ NSCLC which comprises $85 \%$ of all cases of lung cancer was primarily treated with platinum-based chemotherapy prior to the advent of immunotherapy. ${ }^{2}$ Taken as a whole, NSCLC has a 5-year survival rate of $18.1 \%$ and portends a poor prognosis. However, 5-year survival rates vary from $49 \%$ to $1 \%$ for Stage IA NSCLC and Stage IV disease, respectively. ${ }^{3}$ Newer therapies targeting activating mutations in EGFR or ALK have shown improved response rates over chemotherapy in the subset of patients with these mutations; however, majority of patients do not have actionable mutations and therefore will not benefit from targeted therapies. Combination of chemotherapy with angiogenesis inhibitor, bevacizumab, has also yielded minimal added benefit. Immunotherapy with checkpoint inhibitors has shown much promise in recent trials in the treatment of advanced NSCLC. ${ }^{4-7}$ These novel drugs stimulate the host immune system to identify tumor cells as foreign, thereby inhibiting its growth. They also relieve inhibition of the immune system that allows tolerance to tumor growth. Examples of these drugs include antibodies against PD-1: Nivolumab and pembrolizumab and antibodies against PD-L1: Atezolizumab and durvalumab. The authors provide a detailed review of the current evidence supporting their use in the management of NSCLC.
Correspondence: Sameep Sehgal Department of Thoracic Medicine and Surgery, Lewis Katz School of Medicine, Temple University, 340I N Broad St, Philadelphia, PA 19130, USA

Email sameep.sehgal@tuhs.temple.edu 


\section{Background}

Immunotherapeutic strategies for the treatment of lung cancer involve a complex interaction between various components of the innate and adaptive immune systems. ${ }^{8}$ The cell types involved include CD8+ T lymphocytes (cytotoxic T-cells), Th1 and Th2 subtypes of CD4+ T lymphocytes (helper T-cells), NK, Treg and macrophages. Each cell type plays a specific role in the immune cascade that ultimately leads to a cytotoxic response against the tumor cells. The adaptive immune system is responsible for the activation of T-cells, which then aids in the destruction of the tumor. This cascade is limited by various checkpoint signals and studies have noted an increased circulating level of checkpoints, such as PDL-1 and CTLA-4, in these patients, indicating an immunologic role in the pathogenesis of lung cancer. ${ }^{9}$ These checkpoints provide a unique opportunity for drugs to play an "immunomodulatory" role, where such signals can be blocked to prevent suppression of T-cell activation and proliferation.

The adaptive immune system plays a critical role in tumor suppression by identifying and destroying tumor cells. They are able to identify tumor cells as "nonself" antigens and distinguish them from "self" antigens. APCs, which are usually comprised of dendritic cells or macrophages identify tumor cells and "present" them to immature T lymphocytes. The APCs bind to the peptide antigen through the MHC receptors on its cell surface. The antigen/MHC complex subsequently binds to the T-cell receptor on the surface of the immature T-cells. Following this interaction, these immature helper (CD4+) and cytotoxic (CD8+) cells are transformed into their activated counterparts (Figure 1) ${ }^{10}$ Activated T-cells produce inflammatory cytokines which then proliferates, amplifying the immune response against the tumor cells. $\mathrm{CD} 4+$ cells activate $\mathrm{B}$ lymphocytes to form antigen-specific antibodies and the $\mathrm{CD} 8+$ cells directly kill the pathogen. A small proportion of the CD4+ cells make up the NK cells and the Treg cells. Other cells from the CD4+ lineage has also been identified such as Th17 and Th9. The differentiation into these lineages depends on specific cytokine signaling and expression of transcription factors.

\section{Mechanism of action}

A key mechanism that aids tumor proliferation is its ability to evade the immune system. ${ }^{11}$ Under normal circumstances,

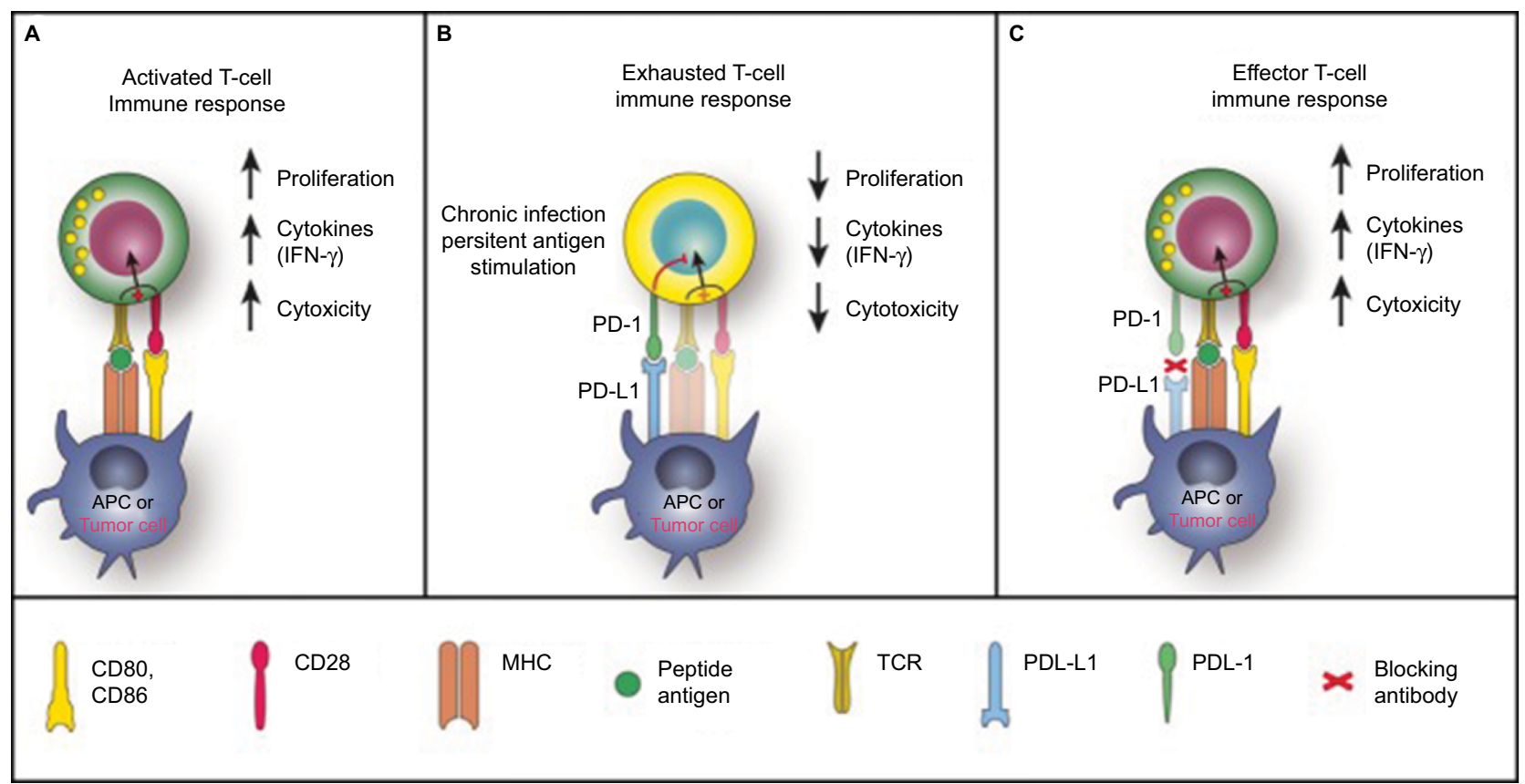

Figure I PD-I in T-cell activation, exhaustion and effector function.

Notes: (A) T-cells are activated via (I) binding of MHC plus peptide on an APC to the TCR and then (2) binding of APC CD80/86 to T-cell CD28. In patients with cancer, tumor cells can also serve as APCs. Upon T-cell activation, PD-I expression is induced. (B) In situations of chronic infection or persistent stimulation, PD-LI signals through T-cell PD-I to "turn off" T-cells in order to minimize damage to healthy tissue. Tumor cells can upregulate PD-LI in order to "turn off" T-cells that might destroy them. (C) Blocking the PD-I/PD-LI signaling pathway allows T-cells to maintain their effector functions. In patients with cancer, activated tumor-specific T-cells can kill tumor cells and secrete cytokines that activate/recruit other immune cells to participate in the antitumor response. Reproduced under Creative Commons Attribution License (CC BY), from McDermott DF, Atkins MB. PD-I as a potential target in cancer therapy. Cancer Med. 20I3;2(5):662-673. (C) 2013 The Authors. Cancer Medicine published by John Wiley. ${ }^{35}$

Abbreviations: APC, antigen-presenting cell; IFN- $\gamma$, interferon gamma; MHC, major histocompatibility complex; PD-I, programmed death-I; PD-LI, PD ligand I; TCR, T-cell receptor. 
a series of checkpoints signals regulate the immune cascade by preventing uncontrolled activation of $\mathrm{T}$ lymphocytes. These checkpoint signals are hardwired into the immune system and comprise the inhibitory pathways that are vital for modulating the amplitude of the immune response as well as for preventing autoimmunity and damage to normal host tissue. One of the main checkpoint molecules that mediate tumor-induced immune suppression is PD-1. PD-1 is a cell surface coinhibitory molecule that is induced on the surface of the activated T-cells and prevents overstimulation of the immune system thereby averting damage to healthy tissues (Figure 1). ${ }^{12}$ PD-L1 is expressed by APCs and is the ligand that binds to PD-1 resulting in inhibition of T-cell receptor signaling and downregulation of antiapoptotic molecules and proinflammatory cytokines. ${ }^{10,13}$ Such receptor/ligand (PD-1/ PD-L1) interactions can be readily blocked by antibodies to these receptors or ligands or alternatively, by recombinant forms of these protein molecules. Tumor cells can express PD-L1 on their cell surface mimicking or stimulating these signals to inhibit the anti-tumor response, thereby helping it to proliferate. This provides a negative feedback to immune activation and results in suppression of T-cell activation. ${ }^{14}$ Checkpoint immunotherapy aims to use highly selective humanized monoclonal antibodies against checkpoint signals such as PD-1 and PD-L1. By blocking these receptors and signals, the immune system can be reactivated to fight the tumor.

\section{Clinical trials in NSCLC}

Several landmark clinical trials have been conducted in patients with advanced NSCLC, with several more currently underway. Clinical trials have primarily been conducted in patients with advanced NSCLC (Table 1).

\section{Nivolumab (Opdivo ${ }^{\circledR}$ )}

Nivolumab (Opdivo; Bristol-Myers Squibb Company, New York, NY, USA) is a fully human IgG4 monoclonal antibody that selectively inhibits PD-1 activity by binding to the PD-1 receptor, thereby blocking the binding of its ligands PD-L1 and PD-L2. ${ }^{15}$

Two RCTs published in 2015, FDA approval of nivolumab as a second-line agent for advanced squamous and nonsquamous NSCLC. Both studies demonstrated a benefit with nivolumab while reducing high-grade adverse effects.

Brahmer et al conducted a Phase III RCT in patients with advanced squamous cell NSCLC, who had disease progression on first-line chemotherapy. ${ }^{5}$ They compared treatment with nivolumab versus docetaxel in 272 patients, with a minimum follow up of 11 months. The OS was significantly longer in the nivolumab group (9.2 months, 95\% CI, 7.3-13.3), compared to the docetaxel group (6.0 months, 95\% CI, 5.1-7.3), and the one-year survival was $42 \%$ in the nivolumab group and $24 \%$ in the docetaxel group. Secondary end-points: response rate (20\% vs 9\%) and median PFS (3.5 months vs 2.8 months) were also improved in the nivolumab group. One-year PFS was $21 \%$ in the nivolumab vs $6 \%$ in the docetaxel group. In subgroups, stratified based on PD-L1 expression ( $1 \%, 5 \%$ and $10 \%$ ), there was an improvement in OS, PFS and ORR across the subgroups, which was similar to patients without PD-L1 expression. Adverse events reported were significantly less in the nivolumab group, although the rate of immune-mediated adverse effects was higher. Grade 3 or 4 adverse effects were lower in the nivolumab group compared to docetaxel (7\% vs $55 \%$ ). Fatigue, asthenia and decreased appetite were the most commonly reported adverse effects.

In the same year, Borghaei et al published an open-label Phase III RCT with 582 patients who had advanced nonsquamous NSCLC with progression on chemotherapy. ${ }^{4}$ Patients were randomized to receive nivolumab or docetaxel. The OS was significantly higher in the nivolumab group: 12.2 months (95\% CI, 9.7-15.0) compared to the docetaxel (9.4 months (95\% CI, 8.1-10.7). The one-year survival was 51\% in the nivolumab group vs 39\% in docetaxel group, and at 18 months OS was $39 \%$ in the nivolumab group vs $23 \%$ in docetaxel group. Other significant end points included a higher ORR ( $19 \%$ for nivolumab vs $12 \%$ for docetaxel) and median DOR (17 months vs 5.6 months) in the nivolumab group. There was no difference in the PFS between the two groups (nivolumab 2.3 months vs docetaxel 4.2 months), with a HR for disease progression or death of 0.92 (95\% CI, $0.77-1.11 ; P=0.39)$. In the pre-specified analysis of PD-L1 expression levels ( $>1 \%, 5 \%$ and $10 \%$ ) nivolumab had a higher OS, PFS and median DOR in the three groups, compared to the docetaxel group. In patients with $<1 \%$ of cells expressing PD-L1, no difference in OS was seen. Significantly less number of patients had grade 3 and 4 adverse effects in the nivolumab group ( $10 \%$ vs $54 \%$ of patients) as well as treatment-related serious adverse effects ( $7 \%$ vs $20 \%)$. The common treatment-related adverse events reported were fatigue, nausea, and asthenia.

\section{Pembrolizumab (Keytruda ${ }^{\circledR}$ )}

Pembrolizumab (Keytruda, Merck Sharp \& Dohme Corp, Kenilworth, NJ, USA) is a highly selective anti-PD-1 humanized monoclonal antibody that inhibits PD-1 activity by binding to the PD-1 receptor on activated T-cells. Three large, multicenter Phase I and II clinical trials were reported in 2016, exploring the use of pembrolizumab as a first-line and second-line treatment in advanced NSCLC. 


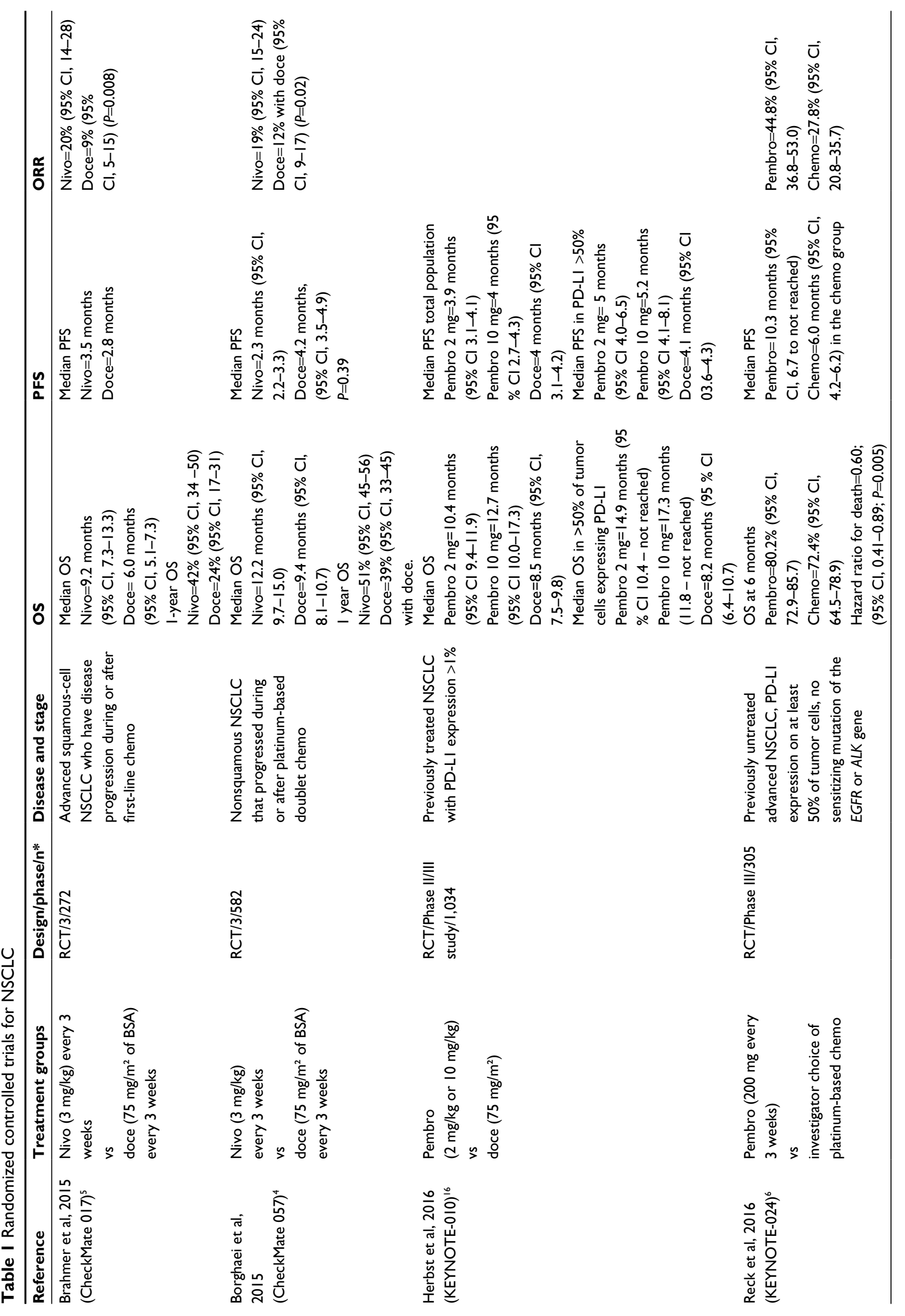




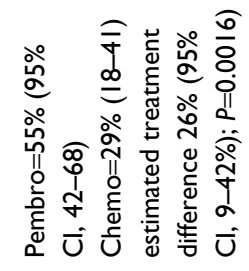

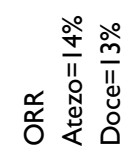
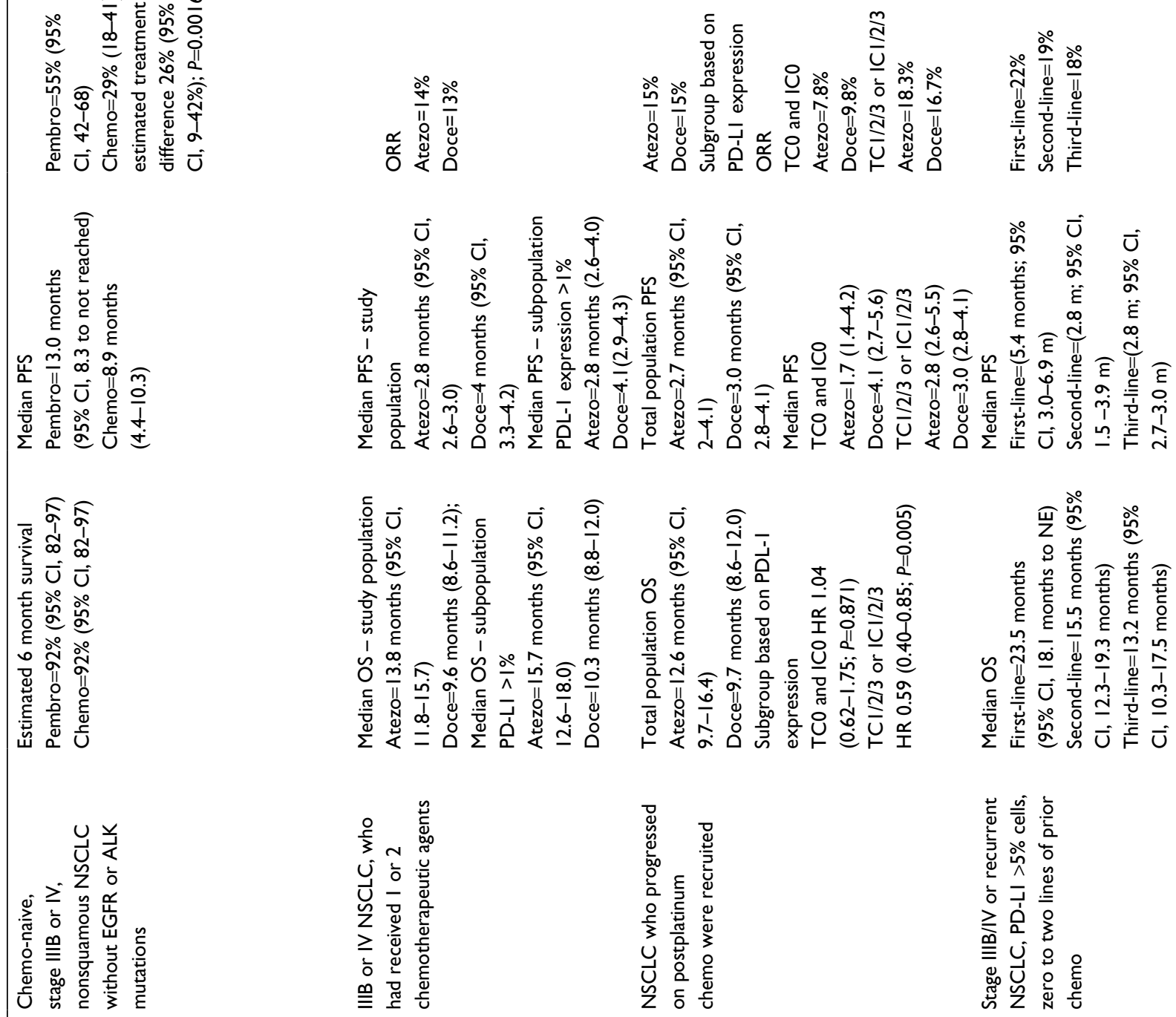

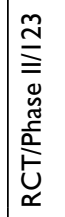

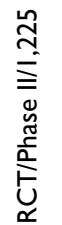

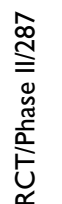
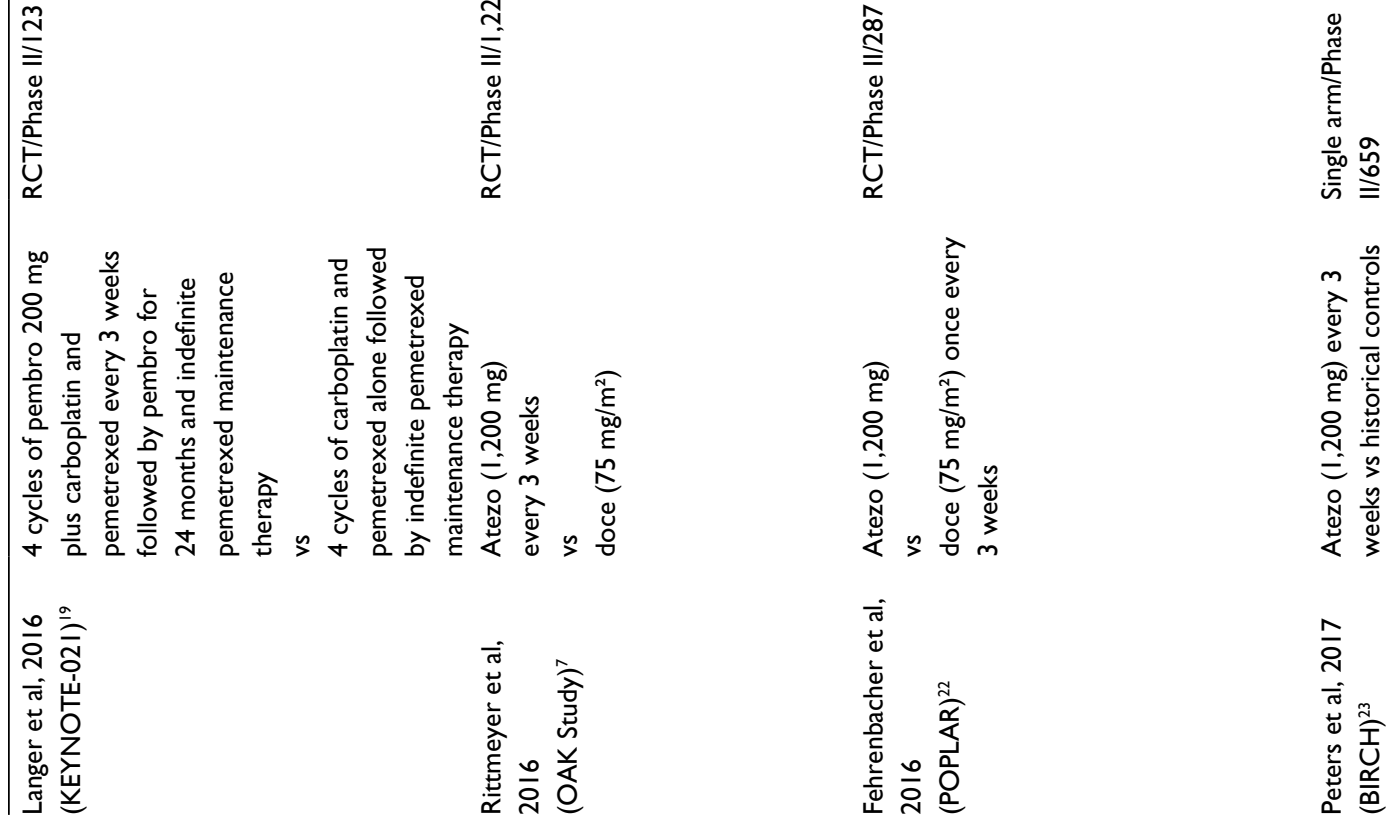


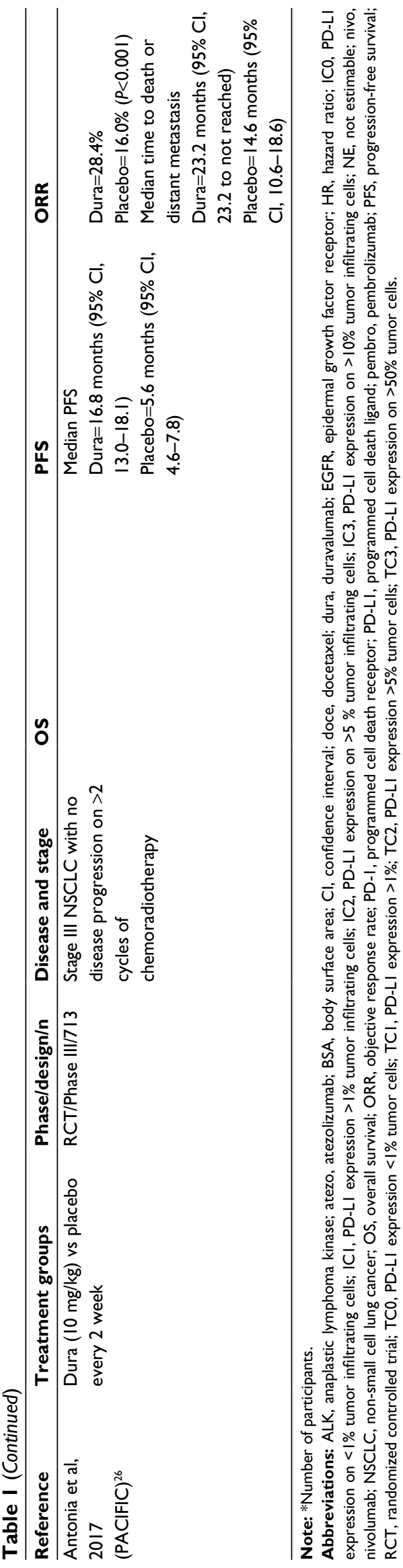

Treatment with pembrolizumab was studied in a Phase II/III RCT in patients with previously treated advanced NSCLC. ${ }^{16}$ In this study, 1,034 patients with PD-L1 expression $>1 \%$ were randomized into three groups to be treated with pembrolizumab ( $2 \mathrm{mg} / \mathrm{kg} / \mathrm{dose}$ or $10 \mathrm{mg} / \mathrm{kg}$ dose $)$ or docetaxel. The primary end points in this study were OS and PFS in the total population and subpopulation with greater than 50\% PD-L1 expression. The OS in both the pembrolizumab $2 \mathrm{mg} / \mathrm{kg}$ (median OS 10.4 months) and 10 $\mathrm{mg} / \mathrm{kg}$ (median OS 12.7 months) groups was significantly longer than the docetaxel group (median OS 8.5 months). PFS was not statistically different between the three groups (3.9 months with pembrolizumab $2 \mathrm{mg}$ vs 4 months with pembrolizumab $10 \mathrm{mg}$ vs 4 months with docetaxel). This lack of PFS benefit to immunotherapy in the overall population may be related in part due to a recently reported mechanism of disease progression in patients with NSCLC who receive PD-1/PD-L1 therapy known as hyperprogressive disease, which can be seen in up to $9 \%$ of patients according to one study. ${ }^{17}$ However, in the subgroup analysis patients with $\geq$ $50 \%$ of tumor cells expressing PD-L1, the OS as well as PFS were higher in the pembrolizumab groups compared to the docetaxel group, suggesting that PD-L1 expression is a predictor of OS and PFS in the second-line setting. Compared to the docetaxel group, the HR for OS was 0.54 (95\% CI, 0.38-0.77; $P=0.0002$ ), in the $2 \mathrm{mg} / \mathrm{kg}$ dose group and $0.50(95 \% \mathrm{CI}, 0.36-0.70 ;<0.0001)$ for the $10 \mathrm{mg} / \mathrm{kg}$ group. Median PFS was longer in the two pembrolizumab groups (5 and 5.2 months) compared to docetaxel group (4.1 months). Overall, efficacy on pembrolizumab was greater in this subpopulation of patients. There was no difference in the OS or PFS between the two pembrolizumab dosing groups in the total or high PD-1 expression subpopulation. Treatment-related adverse effects of grade 3 or higher were lower in the two pembrolizumab groups $(2 \mathrm{mg} / \mathrm{kg}-13 \%$ and $10 \mathrm{mg} / \mathrm{kg}-16 \%$ ) compared to docetaxel group (35\%). Deaths attributed to treatment were seen in 2 patients on the $2 \mathrm{mg} /$ $\mathrm{kg}$ dose, 3 patients in the $10 \mathrm{mg} / \mathrm{kg}$ dose and 5 patients on docetaxel. Immune-mediated adverse effects were common in patients on pembrolizumab ( $19 \%$ patients on $2 \mathrm{mg} / \mathrm{kg}$ and $20 \%$ on $10 \mathrm{mg} / \mathrm{kg}$ ).

Reck et al explored the use of pembrolizumab as monotherapy for the first-line treatment of advanced NSCLC with a high proportion of PD-L1 expression in an open-label Phase-III RCT. ${ }^{6}$ In this study, 305 patients with treatment naïve, stage IV NSCLC, negative for actionable EGFR and ALK mutations, and a PD-L1 expression of $\geq 50 \%$ were randomized to receiving pembrolizumab or investigator's choice 
chemotherapy. The primary end point of PFS was significantly higher in the pembrolizumab group at 10.3 months $(95 \% \mathrm{CI}$, 6.7 to not reached) compared to 6 months (95\% CI, 4.2-6.2) in the chemotherapy group (with a HR of 0.50 for progression or death). Important secondary end points included OS and ORR which were both higher in the pembrolizumab group. Estimated percentage of patients who were alive at 6 months was $80.2 \%(95 \%$ CI, 72.9-85.7) in the pembrolizumab group vs $72.4 \%(95 \% \mathrm{CI}, 64.5-78.9)$ in the chemotherapy group. Objective response was seen in $44.8 \%$ of patients in the pembrolizumab and $27.8 \%$ of patients in the chemotherapy group. Treatment-related grade 3 or higher adverse effects were lower in the pembrolizumab group compared to chemotherapy group (26.6\% vs $53.3 \%$ ), with a higher rate of discontinuation in the chemotherapy group ( $7.1 \%$ vs $10.7 \%)$. Immune-mediated adverse effects were seen in $29 \%$ of patients in the pembrolizumab and $4.7 \%$ of the chemotherapy group. Updated results with a median follow up of 25.2 months, were presented as an abstract at the WCLC in 2017, pembrolizumab group continued to have an improved OS (30.0 (18.3-not reached) compared to $14.2(9.8-19.0)$ months in the chemotherapy group. The estimated survival at 12 months was $72.4 \%$ (95\% CI, 64.5-78.9) in the pembrolizumab group and 54.8\% (95\% CI, 46.4-62.4) in the chemotherapy arm. ${ }^{18}$

Langer et al conducted a Phase II RCT exploring the addition of pembrolizumab to chemotherapy as a first-line agent in patients with advanced NSCLC. ${ }^{19}$ A total of 123 patients with stage IIIB or IV NSCLC, who were negative for actionable EGFR and ALK mutations, were randomized to receive standard chemotherapy alone (carboplatin and pemetrexed, followed by indefinite pemetrexed maintenance) or standard chemotherapy plus pembrolizumab. A significantly higher proportion of patients in the pembrolizumab group (55\% [95\% CI, 42-68]) had an objective response compared to chemotherapy alone group (29\% [95\% CI, 18-41]), with an estimated treatment difference of $26 \%$. None of the patients in either group had a complete response. A higher PFS was seen in the pembrolizumab plus chemotherapy group (13 months vs 8.9 months), but no difference in OS was seen between the two groups (HR 0.90 [95\% CI, 0.42-1.91]; $P=0.39$ ). In subgroups based on PD-L1 expression, patients on pembrolizumab and chemotherapy, with TPS $<1 \%$ had a response rate of $57 \%$, in patients with a TPS of $1-49 \%$ : the response was seen in $26 \%$, and TPS of $>49 \%$ : response was seen in $80 \%$ of the patients. While a higher response rate was seen in TPS score of $>49 \%$, the authors noted that the number of patients in the individual subgroups were too small to draw any conclusion. The incidence of grade 3 or higher adverse effects was higher in the pembrolizumab plus chemotherapy group vs chemotherapy group alone (39\% vs $26 \%$ ). The rate of immune-mediated adverse effects was significantly higher in the pembrolizumab group compared to the chemotherapy alone group (22\% vs $11 \%$ ). Despite having a higher rate of adverse events, the rate of discontinuation of treatment due to adverse effects was similar between the two groups. In the recently presented follow up data by Borghaei et al with a median follow up of 18 months, improved ORR (57\% vs $32 \%)$ and improved PFS (19.0 [8.5-not reached] months vs 8.9 [6.2-11.8]) persisted in the pembrolizumab group. OS was also significantly improved in the pembrolizumab group, with a HR of $0.59(95 \% \mathrm{CI}, 0.34-1.05 ; P=0.034) .{ }^{20}$

\section{Atezolizumab (Tecentriq ${ }^{\circledR}$ )}

Atezolizumab (Tecentriq, Genentech USA, Inc, San Francisco, CA, USA) is a monoclonal antibody that binds to PD-L1 and blocks its interaction with PD-1, thereby enhancing T-cell activity against tumor cells. ${ }^{21}$

Fehrenbacher et al conducted an open-label Phase II RCT, in patients with advanced NSCLC who progressed on firstline chemotherapy. ${ }^{22}$ In this trial, 287 patients were enrolled and were randomized to receive atezolizumab or docetaxel every 3 weeks. OS was longer in the atezolizumab group compared to docetaxel group (12.6 months [95\% CI, 9.7-16.4] vs 9.7 months [95\% CI, 8.6-12]) with a HR of 0.73 (95\% CI, 0.53-0.99). There was no difference in PFS (2.7 months with atezolizumab vs 3 months with docetaxel) or ORR (15\% in both groups). The patients were divided into subpopulations based on PD-L1 expression on tumor cells (TC0: $<1 \%$ expression, TC1: $1-5 \%$ expression, TC2: 5-50\% expression, TC3: $>50 \%$ expression) and tumor-infiltrating immune cells (IC0: $<1 \%$, IC1: 1-5\%, IC2: 5-10\%, IC3: >10\%). In the TC0 and IC0 subgroup, the OS was similar between the atezolizumab and docetaxel groups. As the expression of PD-L1 increased, increasing survival benefit was seen. In the combined TC2/3 and IC 2/3 groups, the atezolizumab showed improved OS, with a HR of $0.54(95 \% \mathrm{CI}, 0.33-0.89 ; P=0.014)$. PFS and ORR were improved in the TC3/IC3 subgroup (PFS HR 0.60 [95\% CI, 0.31-1.16] and ORR 38\% vs 13\%). The other subgroups showed no difference in these parameters. Grade 3 and 4 adverse effects were seen in $11 \%$ patients in the atezolizumab group and $39 \%$ in the docetaxel group. Pneumonitis and increased liver function tests were the most common adverse effects in the atezolizumab group.

The OAK trial was a Phase III open-label RCT that looked into atezolizumab as a second or third-line treatment agent, in patients with advanced NSCLC. ${ }^{7}$ 1,225 patients were 
randomized to treatment with atezolizumab or docetaxel, 950 patients were included in the primary efficacy analysis . The primary end point of median OS was significantly higher in the atezolizumab group compared to the docetaxel group (13.8 months [95\% CI, 11.8-15.7] vs 9.6 months [8.6-11.2]). Secondary end points of PFS (median PFS $=2.8$ months $[95 \%$ CI, 2.6-3] vs 4 months [95\% CI, 3.3-4.2]) and ORR (14\% vs $13 \%$ ) were similar in the two treatment groups. The study population was subdivided based on PD-L1 expression, into expression of $<1 \%$ tumor or tumor-infiltrating immune cells (TC0/IC0) or $>1 \%$ cells (TC1/2/3 and IC $1 / 2 / 3$ ). In both these subpopulations, the OS was higher in the atezolizumab groups compared to docetaxel groups. PFS and ORR were similar in the two treatment arms irrespective of the PD-L1 expression. Grade 3 or 4 adverse events were lower in the atezolizumab groups compared to docetaxel group (37\% vs $54 \%$ of patients). Pneumonitis, hepatitis and colitis were the most common immune-mediated adverse effects in the atezolizumab group. Only $8 \%$ of the patients in the atezolizumab group discontinued treatment due to treatment-related adverse effects compared to $19 \%$ in the docetaxel group.

The BIRCH trial was a Phase II, single-arm study, which looked at treatment of advanced NSCLC with PD-L1 expression $>5 \%$, with atezolizumab monotherapy as first, second or third- line treatment. 659 patients were enrolled, of which 139 received it as first-line treatment (cohort 1), 268 had received first-line chemotherapy (cohort 2) and 252 patients had received at least two prior chemotherapy courses (cohort 3 ). The primary end point was ORR, which was reported as $22 \%$ in cohort $1,19 \%$ in the cohort 2 and $18 \%$ in cohort 3 treatment groups. In the subgroup with the highest PDL-1 expression, the ORR was higher at $20 \%, 32 \%$ and $31 \%$, in cohort 1, 2 and 3, respectively. The secondary end point of OS at a follow up of 22.5 months, was reported at 23.5 months in cohort $1,15.5$ months in cohort 2 and 13.2 months in cohort 3. The OS was similar in the higher PD-L1 subgroup. The authors compared the OS to the historical control group and reported an improvement when atezolizumab was used as a first-line agent (23.5 months vs 10-12 months). ${ }^{23}$

Initial data from the IMpower150 trial was presented at the ESMO immune congress in December 2017. This is a Phase III trial which evaluated the addition of atezolizumab to chemotherapy in treatment naïve patients with EGFR and ALK-negative advanced NSCLC. The data presented reported a comparison between two arms - a combination of atezolizumab, Bevacizumab (arm B) with 356 patients and chemotherapy vs bevacizumab plus chemotherapy (arm C) with 336 patients. The median PFS in the atezolizumab arm was 11.3 months $(95 \% \mathrm{CI}, 9.1-13.0)$ compared to 6.8 months (95\% CI, 5.9-7.4 months) in the control arm. The HR for PFS was reported as $0.62(95 \% \mathrm{CI}, 0.52-0.74 ; P<0.0001){ }^{24}$

\section{Durvalumab (Imfinzi $\left.{ }^{\circledR}\right)$}

Durvalumab (Imfinzi, AstraZeneca, Cambridge, UK) is a human IgG1 $\kappa$ monoclonal antibody that blocks the interaction of PD-L1 with the PD-1 and CD80 (B7.1) molecules. ${ }^{25}$

Antonia et al randomized 709 patients with unresectable Stage III NSCLC who did not progress after 2 or more cycles of definitive chemoradiation to receive the PD-L1 antibody, durvalumab (at a dose of $10 \mathrm{mg} / \mathrm{kg}$ ) or placebo every 2 weeks for up to 12 months. ${ }^{26}$ The study drug was administered from 1-42 days after the patients had received chemoradiotherapy. Their PD-L1 status was typically $<25 \%$ or unknown. The median PFS was 16.8 months $(95 \% \mathrm{CI}$, 13.0-18.1) with durvalumab vs 5.6 months $(95 \% \mathrm{CI}$, 4.6-7.8) with placebo (HR for progression or death, 0.52; 95\% CI, $0.42-0.65 ; P<0.001)$. At 18 -month PFS rate was $44.2 \%$ in patients who received durvalumab vs $27 \%$ in those who received placebo.

\section{Comparison between nivolumab and pembrolizumab}

There have been no studies comparing different immunotherapy agents in patients with advanced NSCLC. A metaanalysis indirectly compared treatment with pembrolizumab and nivolumab in advanced NSCLC. ${ }^{27}$ The authors found no difference in ORR ([OR]: 1.14, 95\% CI, 0.60-2.01), OS (HR: $0.98,95 \%$ CI, 0.35-2.74) or PFS (HR: 1.12, 95\% CI, 0.70-1.77) between treatment with pembrolizumab and nivolumab. Comparing the adverse effect profiles of the two medications, they found a higher rate of grade 3 adverse effects in the pembrolizumab patients compared to patients treated with nivolumab (OR: 3.44, 95\% CI, 1.87-6.32). Immune-mediated adverse events were not different between the two drugs. The incidence of pneumonitis of any grade (OR: $0.25,95 \% \mathrm{CI}, 0.03-1.74$ ) and grade 3 or higher (OR 1.12 (95\% CI, 0.10-12.06), were similar when comparing the two.

Pillai et al conducted a meta-analysis comparing PD1 (nivolumab and pembrolizumab) and PD-L1 inhibitors (atezolizumab, durvalumab and Avelumab) used in the treatment of NSCLC patients. Their analysis included 23 studies which included 5,744 patients who were evaluated for toxicities. There was no difference in the PD1 and PD-L1 groups in the ORR ( $19 \%$ vs $18.6 \% ; P=0.17)$. They did not find any significant difference in the incidence of overall adverse effects $(64 \%$ vs $66 \% ; P=0.8)$, grade $3-5$ adverse 
effects $(13 \%$ vs $21 \% ; P=0.15)$ or immune-related adverse effects ( $16 \%$ vs $11 \% ; P=0.07)$. The incidence of pneumonitis was higher in the PD-1 group compared to the PD-L1 group ( $4 \%$ vs $2 \%, P=0.01){ }^{28}$

\section{Guidelines and regulatory approvals}

Based on these clinical trials, regulatory authorities have approved the use of nivolumab, pembrolizumab and atezolizumab in patients with advanced NSCLC (Tables 2 and 3).

\section{Indications Stage IV NSCLC}

Pembrolizumab is the only immunotherapeutic agent currently approved by the FDA for the first-line treatment of advanced (metastatic/Stage IV) NSCLC. As a single agent, pembrolizumab is approved for patients with advanced NSCLC with PD-L1 expression of at least 50\%. ${ }^{6}$ Pembrolizumab is currently also approved in the first-line setting, in combination with carboplatin and pemetrexed, for previously untreated patients with advanced nonsquamous NSCLC irrespective of PD-L1 expression. ${ }^{19}$ atezolizumab and nivolumab have been approved for second-line treatment of advanced NSCLC irrespective of tumor PD-L1 expression in patients who have progressed after prior platinum-based chemotherapy.

These findings are currently being explored further for confirmation in an international, double-blind, Phase III RCT.
Until these results are confirmed in the Phase III setting, providers may choose to exercise caution when acting on the results of this Phase II data, as first-line chemotherapy can be followed in the second-line by pembrolizumab with PD-L1 level of at least $1 \%$, or atezolizumab/nivolumab irrespective of PD-L1 status.

\section{Stage III NSCLC}

NCCN guidelines recommended the addition of durvalumab for up to 12 months as consolidation therapy in patients with unresectable stage III NSCLC with no disease progression after at least 2 cycles of chemoradiation.

\section{Choice of immunotherapy}

For patients with advanced NSCLC without targetable mutations, using single agent immunotherapy as first-line therapy is dependent on PD-L1 status. If the PD-L1 expression is $\geq$ $50 \%$, then pembrolizumab may be chosen for first-line therapy. atezolizumab and nivolumab are not currently approved for first-line therapy regardless of PD-L1 status, and pembrolizumab is not approved in patients with PD-L1 tumor expression of $<50 \%$. In patients with stage IV NSCLC who are intolerant to platinum-based chemotherapy or progress on or after platinum-based therapy, pembrolizumab can be used if tumor expression of PD-L1 is $\geq 1 \%$. Nivolumab and atezolizumab are approved for second-line treatment for stage

Table 2 Regulatory agencies (FDA and EC approvals for checkpoint inhibitors in NSCLC (current as of November I5, 20I7)

\begin{tabular}{|c|c|c|c|c|}
\hline Agent & Disease and biomarker & Single/combination & FDA approval date & EC approval date \\
\hline Pembrolizumab & Previously untreated metastatic NSCLC (first-line) & $\begin{array}{l}\text { Combination with } \\
\text { pemetrexed and } \\
\text { carboplatin }\end{array}$ & May 2017 & $\begin{array}{l}\text { Oct } 2017-\text { Merck } \\
\text { withdraws the } \\
\text { application }\end{array}$ \\
\hline Pembrolizumab & $\begin{array}{l}\text { First-line treatment of patients with metastatic } \\
\text { NSCLC, with PD-LI expression (TPS) } \geq 50 \% \text { as } \\
\text { determined by an FDA-approved test, with no EGFR } \\
\text { or ALK genomic tumor aberrations }\end{array}$ & Single agent & October 2016 & January 2017 \\
\hline Atezolizumab & $\begin{array}{l}\text { Metastatic NSCLC whose disease progressed during or } \\
\text { following platinum-containing chemotherapy. Patients } \\
\text { with EGFR or ALK genomic tumor aberrations should } \\
\text { have disease progression on FDA-approved therapy } \\
\text { for these aberrations prior to receiving Atezolizumab }\end{array}$ & Single agent & October 2016 & Sept 2017 \\
\hline Pembrolizumab & $\begin{array}{l}\text { Metastatic NSCLC whose tumors express PD-LI (TPS } \\
\geq 1 \% \text { ) with disease progression on or after platinum- } \\
\text { containing chemotherapy }\end{array}$ & Single agent & October 2015 & August 2016 \\
\hline Nivolumab & $\begin{array}{l}\text { Metastatic NSCLC with progression on or after } \\
\text { platinum-based chemotherapy. Patients with EGFR or } \\
\text { ALK genomic tumor aberrations should have disease } \\
\text { progression on FDA-approved therapy for these } \\
\text { aberrations }\end{array}$ & Single agent & October 2015 & April 2016 \\
\hline Nivolumab & $\begin{array}{l}\text { Metastatic squamous NSCLC with progression on or } \\
\text { after platinum-based chemotherapy }\end{array}$ & Single agent & March 2015 & July 2015 \\
\hline
\end{tabular}

Abbreviations: FDA, US Food and Drug Administration; EC, European Commission; NSCLC, non-small cell lung cancer; PD-LI, programmed cell death ligand; EGFR, epidermal growth factor receptor; ALK, anaplastic lymphoma kinase; TPS, tumor proportion score. 
Table 3 Adaption of the American College of Clinical Oncology Guidelines 2017

\begin{tabular}{lllll}
\hline Cancer & Line of treatment & $\begin{array}{l}\text { EGFR/ALK/ROS } \\
\text { gene mutations }\end{array}$ & TPS & Agent \\
\hline Stage 4 NSCLC - squamous cell & First & Negative & $>50 \%$ & Single agent pembrolizumab \\
Stage 4 NSCLC - nonsquamous cell & First & Negative & $>50 \%$ & $\begin{array}{l}\text { Single agent pembrolizumab } \\
\text { Stage 4 NSCLC }\end{array}$ \\
$\begin{array}{llll}\text { Second (received first-line } \\
\text { chemotherapy) }\end{array}$ & Negative & $>1 \%$ & $\begin{array}{l}\text { Single-agent nivolumab, } \\
\text { pembrolizumab or atezolizumab } \\
\text { Stage 4 NSCLC }\end{array}$ \\
& $\begin{array}{l}\text { Second (received first-line } \\
\text { chemotherapy) }\end{array}$ & Negative & $<1 \%$ or unknown & $\begin{array}{l}\text { Single-agent nivolumab or } \\
\text { atezolizumab }\end{array}$ \\
\hline
\end{tabular}

Notes: Data from Hanna et al. ${ }^{36}$

Abbreviations: EGFR, epidermal growth factor receptor; ALK, anaplastic lymphoma kinase; ROS, reactive oxygen species; TPS, tumor proportion score; NSCLC, non-small cell lung cancer.

IV NSCLC irrespective of PD-L1 tumor expression and therefore can be used in patients without any detectable expression of PD-L1. Between the two options, atezolizumab is frequently chosen in this situation presently since dosing every 3 weeks makes it more convenient for patients versus nivolumab which is given every 2 weeks. However, it is important to note that a 4-week dosing schedule for nivolumab has recently been approved by the FDA. Therefore, decision for which agent to use should be based both on efficacy and convenience for the patient. Based on the PACIFIC trial, durvalumab may be used as consolidation therapy for up to 12 months in patients with unresectable stage III NSCLC following good response to 2 or more cycles of chemoradiation. ${ }^{26}$

\section{Adverse reactions associated with PD-I and PDL-I inhibitors \\ Skin rash}

Skin adverse events are among the most common adverse events that occur in patients receiving checkpoint inhibitors and can be seen in up to $34 \%$ of patients who receive PD- 1 or PD-L1 antibodies, which include rash, pruritus or vitiligo. ${ }^{29}$ However, vitiligo is seen more commonly in patients treated for melanoma and rarely if ever seen in patients treated for NSCLC. Severe skin reactions occurred in $3.9 \%$ of patients who received pembrolizumab for NSCLC. ${ }^{6}$ Immune-related skin rash of any grade was seen in up to $9 \%$ of patients who received nivolumab for NSCLC. ${ }^{4}$ For grade 1 skin reactions such as rash and or pruritus, treatment with checkpoint inhibitors can be continued and symptoms can be treated with oral antihistamines, emollients, and mild strength topical steroids. For grade 2 skin reactions, treatment can be continued but the patient should be checked weekly for improvement. If not improved, treatment can't be interrupted until reaction decreased to grade 1 . Moderate to high strength topical steroids should be used. For grade 3 skins reactions, treatment should be interrupted until symptoms revert to grade 1 and high strength topic steroids should be used in addition to topical emollients and oral corticosteroids. For grade 4 reactions, treatment should be held, and patients should be admitted immediately and monitored closely. Treatment consists of intravenous steroids and steroids should be tapered when the reaction resolves. ${ }^{30}$

\section{Pneumonitis}

Symptomatic pneumonitis can occur in 2-7\% of patients with NSCLC treated with PD-1 or PD-L1 antibodies. ${ }^{17}$ Symptomatic pneumonitis may be associated with shortness of breath, chest pain, hypoxia, increased sputum production, fevers or hemoptysis and typical bronchoscopic findings including diffuse lymphocytic infiltrates on biopsy and brushings. Pneumonitis can occasionally present as focal consolidation. ${ }^{31}$ High-dose corticosteroids can induce resolution of symptoms in most patients although radiographic resolution may usually lag behind. In addition to intravenous steroids, Infliximab should be used in the setting of grade 3-4 pneumonitis if there is no improvement or worsening in 48 hours. ${ }^{30}$

\section{Colitis}

Colitis with PD-1 and PD-L1 antibodies are rare. Colitis of any grade was reported in only $3 \%$ of patients in KEYNOTE-024, with up to $2 \%$ having grade $3-4$ toxicity. ${ }^{6}$ Colitis typically occurs at 4-6 weeks of initiating therapy and can take up to 6 weeks for complete resolution with obstruction or perforation as potential complications. A recent study of PD-1 inhibitor-associated gastrointestinal toxicity reported that $87.5 \%$ of patients responded to corticosteroids. ${ }^{32}$ When diarrhea does not improve within 3 days of starting high-dose steroids or recurs during a steroid taper, infliximab should be used.

\section{Hepatitis}

Hepatitis is rarely seen in patients receiving PD-1 and PD-L1 inhibitors. In the OAK trial, hepatitis was only seen in 2 of the 609 patients in the safety analysis (both were grade 3 ). ${ }^{7}$ 


\section{Immune-related endocrinopathies}

Thyroid gland disorders

Both hyper- and hypothyroidism have been described with immune checkpoint inhibitors. Hyperthyroidism is frequently transient and tends to precede the development of hypothyroidism. The PD-1 inhibitors, nivolumab and pembrolizumab, and the PD-L1 inhibitor, atezolizumab, have been associated with thyroid dysfunction rate of $5-10 \%$, irrespective of tumor site of origin. ${ }^{18}$ Thyroid replacement therapy is usually long-lasting.

\section{Hypophysitis}

Hypophysitis is a potentially life-threatening treatmentrelated adverse event which is caused by inflammation of the anterior pituitary. This adverse event is more commonly seen with CTLA-4 inhibitors or combination of CTLA-4 and PD-1 antibodies. However, hypophysitis is very rare in patients with single-agent anti-PD-1 or anti-PD-L1. ${ }^{18}$ In the event of persistent headaches, visual disturbance such as blurring of vision or bitemporal hemianopsia, emergent MRI brain should be performed. Thyroid hormones, LH, FSH, cortisol and ACTH stimulation testing should be performed. High-dose steroids should be initiated and endocrinology should be consulted.

\section{Rheumatological adverse reactions}

Mild to moderate myalgias and arthralgias can occur in $2-12 \%$ of patients; more commonly seen with PD-1 antibodies. ${ }^{33}$ For mild to moderate symptoms, acetaminophen and/or NSAIDs can be used. However, for more severe symptoms, oral prednisone should be considered. For severe and persistent symptoms, consultation with a rheumatologist should be considered.

\section{Other rare toxicities}

Neurological toxicities have been reported in $1 \%$ of patients who have undergone checkpoint inhibition therapy. However, a recent analysis of 59 trials, involving 9,208 patients reported that neurological side effects from checkpoint inhibition were $3.8 \%$ with CTLA-4 inhibitors, $6.1 \%$ with PD-1 inhibitors and $12 \%$ in patients receiving a combination of anti-CTLA-4 and anti-PD-1 treatment. ${ }^{34}$ Several neurological side effects have been reported, including polyneuropathy, Guillan Barre syndrome, posterior reversible leukoencephalopathy, transverse myelitis, encephalitis and aseptic meningitis. The incidence of cardiac toxicity with checkpoint inhibitors is $<1 \%$. Cardiac adverse events reported include, myocarditis, pericarditis, arrhythmias and cardiomyopathy. Nephrotoxicity with checkpoint inhibitors is also very rare, occurring in $<1 \%$ of patients. Hematological toxicities of checkpoint inhibitors are very rare and so far reports include the development of aplastic anemia, autoimmune hemolytic anemia and immune thrombocytopenic purpura.

\section{Conclusion}

Immune checkpoint inhibitors have heralded a new era in the treatment of NSCLC. They offer a treatment option with increased efficacy and fewer high-grade adverse effects to patients with advanced disease. Several clinical trials are exploring the use of checkpoint inhibitors at various stages of small cell lung cancer and NSCLC, as a part combination or single treatment. As of December 2017, over 250 clinical trials are listed on www.clinicaltrials.gov for treatment of lung cancer with pembrolizumab, nivolumab or atezolizumab. While it is likely that the indications of immune checkpoint inhibitors will expand in the future, it is important to note that current regulatory approval is only for patients with advanced NSCLC patients, and in case of pembrolizumab only in combination with a PD-L1 assay. Specifically, pembrolizumab is approved as first-line therapy for advanced NSCLC with PD-L1 levels $\geq 50 \%$ and second-line therapy with PD-L1 expression $\geq 1 \%$. Atezolizumab and nivolumab are approved as second-line therapy irrespective of PD-L1 status.

Immune checkpoint inhibitors also come with a unique set of "immune-mediated" adverse effects, which were not seen with chemotherapy. Immune-mediated adverse effects can lead to a wide range of clinical conditions and can involve every organ system. Common toxicities have included skin rash, pneumonitis, endocrinopathies, colitis, and hepatitis, although toxicity of almost every organ system has been reported. Thus, a high clinical suspicion for potential immunerelated toxicities needs to be maintained with patients on these medications. Treatment of the immune-mediated adverse effects may involve prolonged courses of glucocorticoids, which is a risk factor for PJP infections. Thus, PJP prophylaxis should be considered in patients who require more than $20 \mathrm{mg}$ prednisone for $>4$ weeks or have other disease states/ medications which may compromise immunity.

Immunotherapy with checkpoint inhibitors for advanced NSCLC have resulted in a new paradigm of treatment options resulting in improved OS, and a less severe yet different toxicity profile when compared to chemotherapy. Further studies are needed to determine appropriate sequencing and combination of chemotherapy with immunotherapy, biomarkers for response and to predict toxicities and determination of the causes of primary or secondary resistance to 
immunotherapy. At present, with rapidly increasing use and indications in patients with lung cancer, knowledge about the indications, efficacy and adverse effects is essential not only for oncologists and pulmonologists, but for all healthcare providers taking care of these patients in an inpatient or ambulatory setting.

\section{Abbreviations}

ACTH, adrenocorticotropic hormone; ALK, anaplastic lymphoma kinase; APC, antigen-presenting cell; CI, confidence interval; CTL4, cytotoxic T-lymphocyte-associated antigen 4; DOR, duration of response; EGFR, epidermal growth factor receptor; FDA, US Food and Drug Administration; FSH, follicle-stimulating hormone; HR, hazard ratio; IgG4, immu-

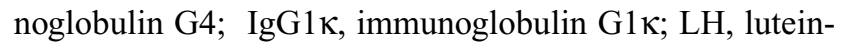
izing hormone; NK, natural killer; NSCLC, non-small cell lung cancer; OR, odds ratio; ORR, objective response rate; OS, overall survival; PD-1, programmed cell death receptor; PD-L1, programmed cell death ligand; PFS, progression-free survival; RCT, randomized controlled trial; Th1, T-helper 1; Th2, T-helper 2; Th9, T-helper 9; Th17, T-helper 17; Treg, regulatory T-cells; TPS, tumor proportion score.

\section{Author contributions}

All authors contributed toward data analysis, drafting and critically revising the paper, gave final approval of the version to be published, and agree to be accountable for all aspects of the work.

\section{Disclosure}

The authors report no conflicts of interest in this work.

\section{References}

1. American Cancer Society. Key Statistics for Lung Cancer. Available from: https://www.cancer.org/cancer/non-small-cell-lung-cancer/about/ key-statistics.html. Accessed April 29, 2018.

2. Herbst RS, Heymach JV, Lippman SM. Lung cancer. $N$ Engl J Med. 2008; 359:1367-1380.

3. American Cancer Society. Non-Small Cell Lung Cancer Survival Rates, by Stage. Available from: https://www.cancer.org/cancer/non-small-celllung-cancer/detection-diagnosis-staging/survival-rates.html. Accessed April 29, 2018.

4. Borghaei H, Paz-Ares L, Horn L, et al. Nivolumab versus docetaxel in advanced nonsquamous non-small-cell lung cancer. $N$ Engl J Med. 2015;373(17):1627-1639.

5. Brahmer J, Reckamp KL, Baas P, et al. Nivolumab versus docetaxel in advanced squamous-Cell non-small-cell lung cancer. $N$ Engl J Med. 2015;373(2):123-135.

6. Reck M, Rodriguez-Abreu D, Robinson AG, et al. Pembrolizumab versus chemotherapy for PD-L1-positive non-small-cell lung cancer. N Engl J Med. 2016;375(19):1823-1833.
7. Rittmeyer A, Barlesi F, Waterkamp D, et al. Atezolizumab versus docetaxel in patients with previously treated non-small-cell lung cancer (OAK): a phase 3, open-label, multicentre randomised controlled trial. Lancet. 2017;389(10066):255-265.

8. Bradbury PA, Shepherd FA. Immunotherapy for lung cancer. J Thorac Oncol. 2008;3(6):S164-S170.

9. Domagala-Kulawik J. The role of the immune system in non-small cell lung carcinoma and potential for therapeutic intervention. Transl Lung Cancer Res. 2015;4(2):177-190.

10. McDermott DF, Atkins MB. PD-1 as a potential target in cancer therapy. Cancer Med. 2013;2(5):662-673.

11. Hanahan D, Weinberg RA. Hallmarks of cancer: the next generation. Cell. 2011;144(5):646-674.

12. Korman AJ, Peggs KS, Allison JP. Checkpoint blockade in cancer immunotherapy. Adv Immunol. 2006;90:297-339.

13. Keir ME, Butte MJ, Freeman GJ, Sharpe AH. PD-1 and its ligands in tolerance and immunity. Annu Rev Immunol. 2008;26:677-704.

14. Pardoll DM. The blockade of immune checkpoints in cancer immunotherapy. Nat Rev Cancer. 2012;12(4):252-264.

15. Robert C, Long GV, Brady B, et al. Nivolumab in previously untreated melanoma without BRAF mutation. $N$ Engl J Med. 2015;372(4): 320-330.

16. Herbst RS, Baas P, Kim DW, et al. Pembrolizumab versus docetaxel for previously treated, PD-L1-positive, advanced non-small-cell lung cancer (KEYNOTE-010): a randomised controlled trial. Lancet. 2016;387(10027):1540-1550.

17. Champiat S, Dercle L, Ammari S, et al. Hyperprogressive disease (HPD) is a new pattern of progression in cancer patients treated by anti-PD-1/ PD-L1. Clin Cancer Res. 2017;23(8):1920-1928.

18. Brahmer J, Rodríguez-Abreu D, Robinson A, et al. OA 17.06 Updated Analysis of KEYNOTE-024: Pembrolizumab vs Platinum-Based Chemotherapy for Advanced NSCLC With PD-L1 TPS $\geq 50 \%$. J Thoracic Oncol. 2017;12(11):S1793-S1794.

19. Langer CJ, Gadgeel SM, Borghaei H, et al. Carboplatin and pemetrexed with or without pembrolizumab for advanced, non-squamous nonsmall-cell lung cancer: a randomised, phase 2 cohort of the open-label KEYNOTE-021 study. Lancet Oncol. 2016;17(11):1497-1508.

20. Borghaei H, Langer CJ, Gadgeel S, et al. Updated results from KEYNOTE-021 cohort G: a randomized, phase II study of pemetrexed and carboplatin with or without pembrolizumab as first-line therapy for advanced nonsquamous non-small-cell lung cancer. Ann Oncol. 2017;28 (Suppl 5):v605-v649. Abstract: LBA49.

21. Krishnamurthy A, Jimeno A. Atezolizumab: a novel PD-L1 inhibitor in cancer therapy with a focus in bladder and non-small cell lung cancers. Drugs Today (Barc). 2017;53(4):217-237.

22. Fehrenbacher L, Spira A, Ballinger M, et al; POPLAR Study Group. Atezolizumab versus docetaxel for patients with previously treated nonsmall-cell lung cancer (POPLAR): a multicentre, open-label, phase 2 randomised controlled trial. Lancet.2016;387(10030):1837-1846.

23. Peters S, Gettinger S, Johnson ML, et al. Phase II trial of atezolizumab as first-line or subsequent therapy for patients with programmed deathligand 1-selected advanced non-small-cell lung cancer (BIRCH). J Clin Oncol. 2017;35(24):2781-2789.

24. Reck M, Socinski MA, Cappuzzo F, et al. LBA1_PRPrimary PFS and safety analyses of a randomized phase III study of carboplatin + paclitaxel +/- bevacizumab, with or without atezolizumab in 1L non-squamous metastatic nsclc (IMPOWER150). Ann Oncol. 2017;28(Suppl 11): mdx760.002-mdx760.002.

25. FDA Drug Label: Durvalumab. Available from: https://www.accessdata. fda.gov/drugsatfda_docs/label/2017/761069s0001bl.pdf. Accessed December 18, 2017

26. Antonia SJ, Villegas A, Daniel D, et al. Durvalumab after chemoradiotherapy in stage III non-small-cell lung cancer. New Eng J Med. 2017;377(20):1919-1929. 
27. Peng TR, Tsai FP, Wu TW. Indirect comparison between pembrolizumab and nivolumab for the treatment of non-small cell lung cancer: a meta-analysis of randomized clinical trials. Int Immunopharmacol. 2017;49:85-94.

28. Pillai RN, Behera M, Owonikoko TK, et al. Comparison of the toxicity profile of PD-1 versus PD-L1 inhibitors in non-small cell lung cancer: a systematic analysis of the literature. Cancer. 2018;124(2):271-277.

29. Belum VR, Benhuri B, Postow MA, et al. Characterisation and management of dermatologic adverse events to agents targeting the PD-1 receptor. Eur J Cancer. 2016;60:12-25.

30. Haanen JBAG, Carbonnel F, Robert C, et al; ESMO Guidelines Committee. Management of toxicities from immunotherapy: ESMO Clinical Practice Guidelines for diagnosis, treatment and follow-up. Ann Oncol. 2017;28(Suppl 4):iv119-iv142.

31. Sehgal S, Velcheti V, Mukhopadhyay S, Stoller JK. Focal lung infiltrate complicating PD-1 inhibitor use: A new pattern of drug-associated lung toxicity? Respir Med Case Rep. 2016;19:118-120.
32. Collins M, Michot JM, Danlos FX, et al. Inflammatory gastrointestinal diseases associated with PD-1 blockade antibodies. Ann Oncol. 2017;28(11):2860-2865.

33. Spain L, Diem S, Larkin J. Management of toxicities of immune checkpoint inhibitors. Cancer Treat Rev. 2016;44:51-60.

34. Cuzzubbo S, Javeri F, Tissier M, et al. Neurological adverse events associated with immune checkpoint inhibitors: review of the literature. Eur J Cancer. 2017;73:1-8.

35. McDermott DF, Atkins MB. PD-1 as a potential target in cancer therapy. Cancer Med. 2013;2(5):662-673.

36. Hanna N, Johnson D, Temin S, et al. Systemic Therapy for Stage IV Non-Small-Cell Lung Cancer: American Society of Clinical Oncology Clinical Practice Guideline Update. J Clin Oncol. 2017;20;35(30): 3484-3515.
ImmunoTargets and Therapy

\section{Publish your work in this journal}

ImmunoTargets and Therapy is an international, peer-reviewed open access journal focusing on the immunological basis of diseases, potential targets for immune based therapy and treatment protocols employed to improve patient management Basic immunology and physiology of the immune system in health, and disease will be also covered. In addition, the journal will focus on the impact of manage-

\section{Dovepress}

ment programs and new therapeutic agents and protocols on patient perspectives such as quality of life, adherence and satisfaction. The manuscript management system is completely online and includes a very quick and fair peer-review system, which is all easy to use. Visit http://www.dovepress.com/testimonials.php to read real quotes from published authors. 